\title{
Aberrant subclavian: new face of an old disease
}

\author{
Saleem Jahangeer ${ }^{1}$, Mohamad Bashir ${ }^{1}$, Amer Harky ${ }^{1,2}$, John Yap ${ }^{1}$ \\ ${ }^{1}$ Department of Cardiac Surgery, Barts Heart Centre, St Bartholomew's Hospital, West Smithfield, London, UK; ${ }^{2}$ School of Medicine, Cardiff \\ University, Cardiff, UK \\ Contributions: (I) Conception and design: M Bashir, S Jahangeer; (II) Administrative support: A Harky, M Bashir; (III) Provision of study materials or \\ patients: None; (IV) Collection and assembly of data: None; (V) Data analysis and interpretation: None; (VI) Manuscript writing: All authors; (VII) \\ Final approval of manuscript: All authors. \\ Correspondence to: Mohamad Bashir, MD, PhD, MRCS. Department of Cardiac Surgery, Barts Heart Centre, St. Bartholomew's Hospital, London. \\ Email: Mohamad.Bashir@bartshealth.nhs.uk.
}

\begin{abstract}
An aneurysm of an aberrant subclavian artery is un usual prevalence of anomalies of aortic arch, with a literature reported prevalence of $2 \%$. Timely elective intervention is of paramount. The advancement in stenting techniques has promoted this practice to be an alternative to conventional open surgical repair which is associated with high rates of perioperative complications including mortality outcomes. However, new faces of this old disease are emerging as we move to novelty and innovation era. We discuss in this review the advancements in this disease entity highlighting and collecting the world experiences.
\end{abstract}

Keywords: Aberrant subclavian; aortic surgery; interrupted aortic arch; endovascular; hybrid; aneurysm

Received: 13 April 2018; Accepted: 04 May 2018; Published: 22 May 2018.

doi: 10.21037/jovs.2018.05.11

View this article at: http://dx.doi.org/10.21037/jovs.2018.05.11

\section{Introduction}

Despite the recent advent seen in aortic arch surgery and imaging modalities that paved way to further understanding and planning of effective surgery on aberrant subclavian artery, there remain lack of standardised approach. Most of the published data available today rely on different surgical, endovascular, or hybrid techniques lack homogeneous reporting and is very much influenced by heterogeneous data extracted from observational and centre experiences.

By in large, the variability of interventions, population cohorts, anatomical and morphological led to inconsistency and biased outcomes. Given all this, we believe that surgical management is best tailored to every patient and should be performed in high volume centres which have access to resources and expertise enabling them to offer alternative treatment strategies (open, hybrid, or endovascular) in elective and non-elective settings. Thus, the unique anatomic location necessitates special considerations not only concerned with the heart itself, but also with cerebral protection.

Its therefore our aim in this article to review, present objectively and appraise the current modalities employed in surgical management of aberrant subclavian artery.

\section{Anatomical \& morphological variation}

Arguably, aberrant right subclavian artery is the commonest embryological abnormality of the aortic arch; this anomaly is reported in literature to be known as "arteria lusoria". In 1735, Hunauld (1) was the first to describe this anomaly. Later, Bayford in 1787 (2), reported a case of long standing dysphagia which found to have an aberrant right subclavian artery at autopsy; he named this condition as "dysphagia lusoria". Hence, the entity of Bayford-Autenrieth dysphagia was ascribed to this disease and to the pioneers that described this old disease.

Currently, the reported incidence of aberrant subclavian artery in the general normal population is between $0.5-2 \%$ at prenatal and postmortem examinations (3-5). However, this rate could go as high as $100 \%$ in patients with trisomy 21 (6).

In a normal setting, the right subclavian is the first of two branches of innominate artery. however, in an aberrant setting, the innominate artery is missing and four large arteries are coming off the aortic arch: namely, the 


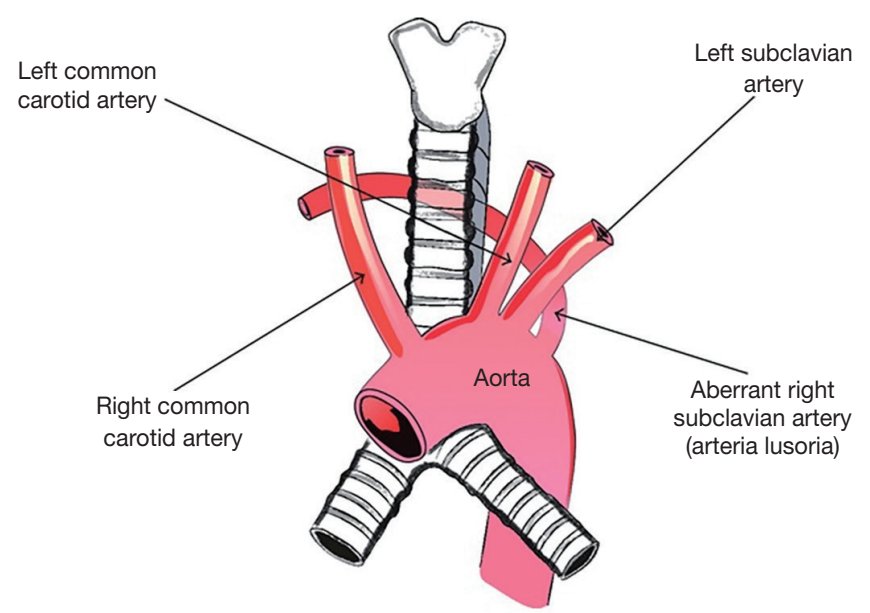

Figure 1 Showing position of the aberrant subclavian artery.

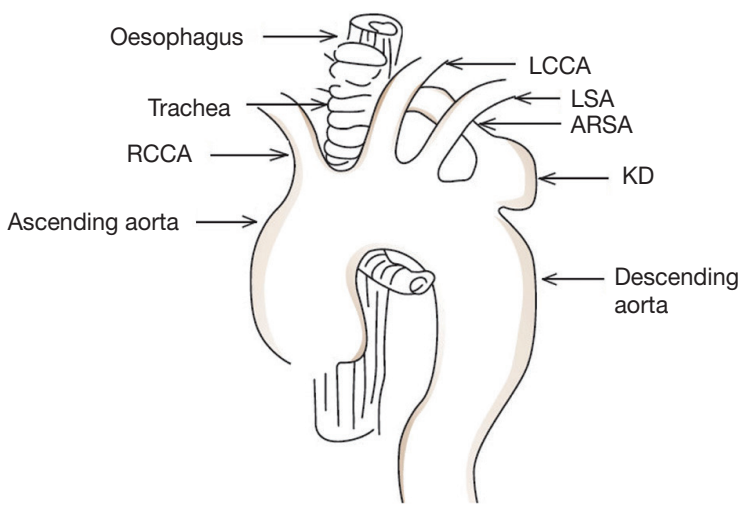

Figure 2 Diagram showing Kommerell diverticulum (KD). ARSA, aberrant right subclavian artery; LSV, left subclavian artery; LCCA, left common carotid artery; RCCA, right common carotid artery.

right common carotid, the left common carotid, the left subclavian with the most distal left sided origin, the right subclavian arteries (Figure 1) which continues to the right arm crossing the middle aspect of the body and passing behind the oesophagus.

It's also been described based on series of autopsy that the aberrant vessel may arise from the first segment of the descending aorta and, passing behind the trachea and cranially as well as rightwards (7). Yet in some instances, the described artery might compress the oesophagus producing a condition known as dysphagia lusoria (8). Kommerell reported (9) a rare case where arteria lusoria originates from an aortic arch diverticulum at the proximal descending aorta leading to the well described Kommerell diverticulum (Figure 2).

\section{How prevalent is aberrant subclavian artery?}

Knowing the prevalence of this anomaly is not only important alone, but rather it helps us to understand the presence of potential other associated vascular anomalies. Presence of such anomalies are dependent on many factors, such as demographics, population sample and the trend in epidemiological changes. Therefore, the frequency of aberrant subclavian artery has been reported to be variable at international level. In United Kingdom, it was reported by Kelly et al. to be of $0.11 \%$ (10), while in Greece the rate was $0.16 \%$ as reported by Natsis et al. (11), $0.3 \%$ in France as reported by Abhaichand et al. (12), and finally $0.38 \%$ in Nederland as reported by de Luca et al. (13). Contrastingly, the rate reported to be different in Asia as of $0.1-0.2 \%$ [Korea; Nie et al. (14) and Japan; Saito et al. (15)]. While the rate in North America reported to be $0.5 \%$ of cases [United State; Haesemeyer and Gavant (16)]; or Australia and Oceania, $0.8 \%$ of cases [New Zealand, Cairney (17)].

This surely dictates the prevalence of this disease with geographical index variation and presentation. It's quite important to have a unified strategy to tackle this aberration and be equipped with background knowledge and experience to dealing with it rendering it safe for the surgeon and patient alike to confront this disease with preconceived awareness and readiness of a plan to execute.

\section{Classification system}

The only classification system for aberrant subclavian presentation was attempted by Kieffer and colleagues in 1994 (18). This indicates that this historical problem has not seen innovation in current multimodality and imaging era. Yet, Kieffer et al. built their classification system on only thirty-three cases with different therapeutic strategies. They proposed classifying patients into four major aberrant RSA groups depending on their anatomical characteristics:

* Group 1: patients with dysphagia caused by nonaneurysmal aberrant RSA;

* Group 2: patients with symptomatic occlusive disease of non-aneurysmal aberrant RSA;

- Group 3: patients with aneurysmal aberrant RSA without aortic lesions, with or without oesophageal compression or arterial thromboembolism;

- Group 4: patients with an aortic (usually aneurysmal) lesion involving the origin of the 
aberrant RSA, with or without aberrant RSA aneurysm.

Having discussed the prevalence of this disease and its equivocal geographic variation and clinical, morphological and demographic changes, the classification system should be recognized and validate accordingly. This will allow wider inclusion and distribution of all population effected. This classification will help choosing type of the approach which in turn relies on the presence or absence of aneurysm and age of the patient.

\section{Should we operate on every patient with aberrant subclavian artery?}

When considering aberrant subclavian artery surgery, one needs to consider multiple factors and a strategy to execute a safe intervention. Since there is a lack of randomised data, guidelines and consensus on this entity, there remain no clear indications or criteria; every case presentation in current era is evaluated based on its own merits and patient selectivity.

\section{Indolent presentation}

It's very likely for aberrant subclavian to have an indolent course. Compression symptoms occurring as a result of arteria lusoria have been reported to only in $7-10 \%$ of adult patients with such anomaly (19). Hence, potentially, the anomaly can remain clinically silent in $90-93 \%$ of cases (20). However, majority of presentation of such anomaly is either in the form of combination with other vascular anomalies or result from compression effect to adjacent structures. This could easily allow aberrant identification, diagnosis and its associated anomalies. Based on the findings, the selection process can be judged not only owing to surgeon volume and experience but also to the anatomy and associated anomalies presented.

\section{Common sense is common}

The most commonly reported compression symptoms as a result of were dysphagia (71.2\%), shortness of breathing (18.7\%), retrosternal pain (17.0\%), cough (7.6\%), and weight loss greater than $10 \mathrm{~kg}$ over a 6-month period (5.9\%) (19). Less common symptoms include; stomach-ache, back pain, and numbness of the right upper limb. The most common vascular anomalies present with an aberrant right subclavian artery were truncus bicaroticus, 19.2\% (27/141);
Kommerell's diverticulum, 14.9\% (21/141); aneurysm after the origin of arteria lusoria (just after the origin of arteria lusoria), 12.8\% (18/141); and finally, right-sided aortic arch, $9.2 \%(13 / 141)$.

\section{Diagnostic sensitivity}

A good knowledge with anatomy and most common types of vascular anomalies associated with aberrant subclavian is of high paramount. This will aid in deciding on patient selection, well organized and structured thoughts for multidisciplinary team meeting outcomes, and a tailored suitable strategy for appropriate intervention. In nowadays imaging modalities, diagnosis and patient selection could be attained by 64 slice computed tomography. According to Yang et al. (21), computed tomography and Doppler studies had a diagnostic sensitivity of $100 \%$ and $97.6 \%$ in detecting aberrant right subclavian artery.

Follow on to this, Chen et al. (22) stated that computed tomography has a $100 \%$ sensitivity in the diagnosis of aberrant subclavian artery, while transthoracic echocardiography has a $92 \%$ sensitivity.

\section{Presence of an aneurysm or dissection}

The scarcity in reporting cases of surgical treatment of aneurysms or aortic dissection associated with an aberrant left subclavian artery is a true translation of how unusual are this condition and the associated technical challenges in managing such anomaly. Those findings are unique to each patient including the pattern of the aortic arch vessels and the relation of aorta to any anomalous originating vessels, however not to forget the relation of trachea and oesophagus to aorta and arch vessels with or without any relative cardiac anomalies. Endovascular, hybrid and open aortic arch and subclavian artery repair has been discussed in selected cases and in many observational studies and will be discussed below.

\section{Surgical management}

When dealing with aberrant subclavian, in majority of cases an access through thoracotomy or median sternotomy is needed. Many authors have suggested that there is no need for thoracotomy or extensive dissection, instead advocating a right supraclavicular approach through a single port incision to obtain the desired surgical goals of ligation, and thereafter the re-implantation will suffice $(7,23)$. 


\section{Open surgical repair}

Several surgical approaches have been explored since the first successful effort of Candardjis et al. (24) in 1961. Vascular incompetence and subclavian artery steal syndrome can manifest from subclavian artery interruption and this was clearly described in many case series. Efforts to achieve adequate control of the aneurysm and reconstruction of subclavian flow through a single incision are hampered as the subclavian artery is commonly aneurysmal near the thoracic outlet, whereas proximal control of the aneurysm through a right thoracotomy incision is difficult (25).

In majority of the cases, the oesophagus is adherent to the anterior wall of the aneurysm, and the neck of the aneurysm originates toward the left of the midline and this can be quite challenging. Esquivel and Miller (26) addressed this problem with axillo-axillary bypass using an $8-\mathrm{mm}$ synthetic graft followed by a left posterolateral thoracotomy incision. On the other hand, Campbell (27), reported the first reconstitution of subclavian flow using surgical approach and deployment of intraluminal prosthetic graft.-

Van Son et al. (28) found that the aberrant right subclavian artery necessitates an open surgical approach through a right thoracotomy which enables mobilization of the vessel, dividing it at its base and directly connecting it to the ascending aorta or right common carotid artery. However, Keiffer and colleagues (18) have emphasized such open repairs can be complicated by the presence of certain other co-existing anomalies, those includes: these includes abnormal origin of the right vertebral artery from the aorta or from the right common carotid artery, the presence of a common carotid trunk, a right-sided thoracic duct, and a non-recurrent laryngeal nerve.

Parrott (29) was among the first to report using an endto-side subclavian to carotid anastomosis on the right side using a supraclavicular incision, this technique reported simultaneously by Hallman and Cooley (30) in which the distal end of the subclavian artery was joined to the side of the carotid within the right thoracic cavity.

Understandably, there is more than one established open approach by which successful repair can be achieved, yet, there are several subtleties and considerations to be addressed in each case. Hence, as there is marked anatomical variations and no reliable and valid classification system, a surgeon will opt to prioritize the surgical steps to provide safe procedure and repair through appropriate aneurysm control right from the origin at descending thoracic aorta; however, this technique is associated with variable degree of spinal cord ischaemia due to clamping the distal descending thoracic aorta. Finally, one should bear in mind that such anomaly could be associated with atherosclerotic changes at the origin of the aneurysm and thus a difficult and unsafe access through right thoracotomy.

\section{Endovascular \& hybrid approaches}

The advancement in endovascular technique and use of different catheter-based aortic valve repair, has warranted the dynamic change in managing such type of subclavian artery anomalies and thus improved perioperative outcomes when compared with conventional open repair techniques, this is of particular importance considering the aged population and have multiple comorbidities that deemed high risk for open repair. This ideal method in such cohort involves a combination of debranching the supra-aortic vessels and then stenting the aortic arch and excluding the pathology (31). However, this technique is not free from its drawbacks, it requires a customized manufactured product that matches the patients' anatomy and disease location; therefore many questions has come into practice, such as durability of the prosthesis, ability of the prosthesis to withstand the increased shear force by the origin of supraaortic vessels, and perioperative clinical outcomes in using such prosthesis including long term survival. Nevertheless, in addition to prosthesis issues, understanding the anatomy and the underline pathology, specially the segment close to the anomaly, are crucial to perform hybrid procedures to treat the aberrant artery.

Lacroix et al. has demonstrated that the combined use of endovascular and open surgical treatment of the aneurysmal aberrant subclavian is achievable safe, and reliable; such less invasive approach could be the treatment of choice in highrisk patients (32).

Yet, current literature provides limited data on managing such cases and majority of the reports are limited to case series and ultimately they are inconsistent in their outcomes. There have been modest outcomes reported from the use of combined endovascular and surgical repair in treating such aneurysmal disease with total exclusion of the aberrant subclavian aneurysm $(31,32)$. In a recent study by Hutton et al. (33), he described a case of intentional occlusion of an aberrant subclavian artery during endovascular stent-graft repair of a descending aortic aneurysm; although the patient has developed subclavian steal at early stage, but this has settled a week later. Similarly, Mok et al. reported the occurrence of gangrene and subclavian steal after operations for correction 
of dysphagia lusoria (34). Currently, there are some reports in literature describing hybrid endovascular repair of a Kommerell diverticulum (9), but few reports describe hybrid endovascular repair of an aberrant subclavian combined with an aortic aneurysm or dissection (35). The advantage of hybrid approach is avoidance of cardiopulmonary bypass and hypothermic circulatory arrest which as the reader appreciate is a must for conventional open surgery, this is also coupled with brain protection method.

Previously, correction of such anomaly has be recommended as the fear of upper limb or vertebrobasilar ischaemia was present (18), additionally the risk of aneurysmal dilatation of the orifice and high risk for rupture (19). Hence current series emerged performing right carotid-subclavian bypass and coil embolization of the aberrant subclavian proximal to the vertebral artery after TEVAR procedure to achieve complete thrombosis of the aberrant vessel. Morris et al. (36) occluded the orifice of the aberrant right subclavian artery using an Amplatzer Vascular Plug (St. Jude Medical, St. Paul, MN, USA) and added a carotid subclavian bypass via a supraclavicular incision.

Similarly, Kedora et al. (37) in their study, occluded the aberrant vessel with a Zenith iliac plug (Cook Medical). However, the closer proximity of the anomaly to oesophagus, the higher rate of subclavian-esophageal fistula which can be fatal if not managed properly.

More advancement reported by Gafoor et al. (38). They reported a fully percutaneous management of Kommerell diverticulum under local anaesthesia. Their procedure may be the minimum invasive method among every conceivable procedure and era of innovation. Yet, it remains a striking feature that we ought to entertain, the long-term outcome is unclear.

\section{Conclusions}

Aberrant subclavian artery interventions are emerging with novelty and innovation, however, its remains elusive as to where such innovations can yield. The disease entity lacks structural and systematic understanding, consensus, longterm results and multidisciplinary approval. We are moving away from conventional culprits, but we ought to remember that this is an old disease with multiple new faces perceived in nowadays practice.

\section{Acknowledgements}

None.

\section{Footnote}

Conflicts of Interest: The authors have no conflicts of interest to declare.

\section{References}

1. Hunauld P. Examen de quelques parties d'un singe. Hist Acad Roy Sci 1735;2:516-23.

2. Bayford D. An Account of a Singular Case of Obstructed Deglutition. London, UK: Memoirs of the Medical Society of London; 1787.

3. Gul A, Corbacioglu A, Bakirci IT, et al. Associated anomalies and outcome of fetal aberrant right subclavian artery. Arch Gynecol Obstet 2012;285:27-30.

4. De León-Luis J, Gamez F, Bravo C, et al. Secondtrimester fetal aberrant right subclavian artery: original study, systematic review and meta-analysis of performance in detection of Down syndrome. Ultrasound Obstet Gynecol 2014;44:147-53.

5. Willruth AM, Dwinger N, Ritgen J, et al. Fetal aberrant right subclavian artery (ARSA): a potential new soft marker in the genetic scan? Ultraschall Med 2012;33:E114-8.

6. Song MJ, Han BH, Kim YH, et al. Prenatal diagnosis of aberrant right subclavian artery in an unselected population. Ultrasonography 2017;36:278-83.

7. Atay Y, Engin C, Posacioglu H, et al. Surgical approaches to the aberrant right subclavian artery. Tex Heart Inst J 2006;33:477-81.

8. Rogers AD, Nel M, Eloff EP, et al. Dysphagia lusoria: a case of an aberrant right subclavian artery and a bicarotid trunk. ISRN Surg 2011;2011:819295.

9. Kommerell B. Verlagerung des Osophagus durch eine abnorm verlaufende Arteria subclavia dextra (Arteria lusoria). Fortschr Geb Roentgenstrahlen 1936;54:590-5.

10. Kelly MD. Endoscopy and the aberrant right subclavian artery. Am Surg 2007;73:1259-61.

11. Natsis KI, Tsitouridis IA, Didagelos MV, et al. Anatomical variations in the branches of the human aortic arch in 633 angiographies: clinical significance and literature review. Surg Radiol Anat 2009;31:319-23.

12. Abhaichand RK, Louvard Y, Gobeil JF, et al. The problem of arteria lusoria in right transradial coronary angiography and angioplasty. Catheter Cardiovasc Interv 2001;54:196201.

13. De Luca L, Bergman JJ, Tytgat GN, et al. EUS imaging of the arteria lusoria: case series and review. Gastrointest Endosc. 2000;52:670-3. 
14. Nie B, Zhou YJ, Li GZ, et al. Clinical study of arterial anatomic variations for transradial coronary procedure in Chinese population. Chin Med J (Engl) 2009;122:2097-102.

15. Saito T, Tamatsukuri Y, Hitosugi T, et al. Three cases of retroesophageal right subclavian artery. J Nippon Med Sch 2005;72:375-82.

16. Haesemeyer SW, Gavant ML. Imaging of acute traumatic aortic tear in patients with an aberrant right subclavian artery. AJR Am J Roentgenol 1999;172:117-20.

17. Cairney J. Tortuosity of the cervical segment of the internal carotid artery. J Anat 1924;59:87-96.

18. Kieffer E, Bahnini A, Koskas F. Aberrant subclavian artery: surgical treatment in thirty-three adult patients. J Vasc Surg 1994;19:100-9; discussion 110-1.

19. Polguj M, Chrzanowski L, Kasprzak JD, et al. The Aberrant Right Subclavian Artery (Arteria Lusoria): The Morphological and Clinical Aspects of One of the Most Important Variations-A Systematic Study of 141 Reports. ScientificWorldJournal 2014;2014:292734.

20. Delap TG, Jones SE, Johnson DR. Aneurysm of an aberrant right subclavian artery presenting as dysphagia lusoria. Ann Otol Rhinol Laryngol 2000;109:231-4.

21. Yang $M, M o X$, Jin J, et al. Diagnostic value of 64 multislice CT in typing of congenital aortic anomaly in neonates and infants. Zhonghua Yi Xue Za Zhi. 2010;90:2167-71.

22. Chen X, Qu YJ, Peng ZY, et al. Diagnosis of congenital aortic arch anomalies in Chinese children by multidetector computed tomography angiography. J Huazhong Univ Sci Technolog Med Sci 2013;33:447-51.

23. Kamiya H, Knobloch K, Lotz J, et al. Surgical treatment of aberrant right subclavian artery (arteria lusoria) aneurysm using three different methods. Ann Thorac Surg 2006;82:187-90.

24. Candardjis G, Desbaillets P, Saegesser F. Coarctation simultanee de l'aorte et d'une arteria lusoria avec aneurisme. Radiol Clin (Basel) 1961;30:308-22.

25. Drullinsky D, Gill H, Bayne JP, et al. Hybrid management of a ruptured right subclavian artery aneurysm dissection. J Vasc Surg Cases Innov Tech 2017;3:198-200.

doi: 10.21037/jovs.2018.05.11

Cite this article as: Jahangeer S, Bashir M, Harky A, Yap J. Aberrant subclavian: new face of an old disease. J Vis Surg 2018;4:108.
26. Esquivel CO, Miller GE Jr. Aneurysm of anomalous right subclavian artery. Contemp Surg 1984;24:81-5.

27. Campbell CF. Repair of an aneurysm of an aberrant retroesophageal right subclavian artery arising from Kommerell's diverticulum. J Thorac Cardiovasc Surg 1971;62:330-4.

28. van Son JA, Mierzwa M, Mohr FW. Resection of atherosclerotic aneurysm at origin of aberrant right subclavian artery. Eur J Cardiothorac Surg 1999;16:576-9.

29. Parrott JC. The subclavian steal syndrome. Arch Surg 1964;88:e661-5.

30. Hallman GL, Cooley DA. Congenital aortic vascular ring. Surgical considerations. Arch Surg 1964;88:666-74.

31. Preventza O, Aftab M, Coselli JS. Hybrid Techniques for Complex Aortic Arch Surgery. Tex Heart Inst J 2013;40:568-71.

32. Lacroix V, Astarci P, Philippe D, et al. Endovascular treatment of an aneurysmal aberrant right subclavian artery. J Endovasc Ther 2003;10:190-4.

33. Hutton M, Wolf B, Boyne N, et al. A case report of intentional occlusion of an aberrant right subclavian artery during endovascular stent-graft repair of a descending aortic aneurysm. Eur J Vasc Endovasc Surg 2004;27:222-4.

34. Mok CK, Cheung KL, Kong SM, et al. Translocating the abberant right subclavian artery in dysphagia lusoria. $\mathrm{Br} \mathrm{J}$ Surg 1979;66:113-6.

35. Austin EH, Wolfe WG. Aneurysm of aberrant subclavian artery with a review of the literature. J Vasc Surg 1985;2:571-7.

36. Morris ME, Benjamin M, Gardner GP, et al. The use of the Amplatzer plug to treat dysphagia lusoria caused by an aberrant right subclavian artery. Ann Vasc Surg 2010;24:416.e5-8.

37. Kedora J, Grimsley B, Pearl G. Endovascular treatment of an aberrant right subclavian artery aneurysm with use of the Zenith iliac plug. Proc (Bayl Univ Med Cent) 2009;22:144-5.

38. Gafoor S, Stelter W, Bertog S, et al. Fully percutaneous treatment of an aberrant right subclavian artery and thoracic aortic aneurysm. Vasc Med 2013;18:139-44. 